\title{
Analysis of Factors and Nursing Countermeasures of the Falling of the Psychotic Inpatients
}

\author{
Chen $\mathrm{Li}^{*}$ and Chen Xiao \\ Hospital of No. 8740 People's Armed Force, Nanchong, Sichuan 637000, China
}

\begin{abstract}
Objective: To study and analyze the factors that cause the falling of the psychotic inpatients in order to control and avoid the falling risk of these patients therefore protecting them from the relevant danger to the greatest extent. Method: Collect the tumble cases of the psychotic inpatients of our hospital in this year and analyze the specific factors leading to the fall from perspectives of the patients by themselves and the hospital environment. Result: By comparing the falling cases, it is found out that $82.0 \%$ of the falls were caused by the factors of the patients themselves, which is a higher probability than the fall caused by the hospital environment, which is $18.0 \%$. Among the factors of the patients themselves, illness factor plays a leading role by causing $34.0 \%$ of the falling cases; $59.0 \%$ of the fall occur at midnight and $54.0 \%$ of the fall occur in the toilets. Conclusion: In order to prevent psychotic inpatients from falling, we have to strengthen the monitoring of the illness condition of these patients. The nursing staff should learn and implement the importance of the nursing life for the psychotic patients, and relevant preventive and protective facilities should be built in the psychotic inpatients' environment.
\end{abstract}

\author{
KEYWORDS \\ Mental disease \\ Inpatient \\ Falling \\ Preventive nursing
}

\section{Introduction}

Psychiatry Department is an important clinical department, which treats a variety of diseases including the commonly-seen schizophrenia, Alzheimer Disease and affective disorders, etc. The clinical symptoms of the patients with the aforesaid diseases are significant and most of these patients need hospitalization. Currently, there are many methods that can be used to treat mental illnesses and satisfactory treatment results can be achieved. However, due to lack of ideal nursing methods, the incidence rate of the falling of the inpatients tends to be high [1]. Compared to the sane patients, the psychotic patients lack self-care ability during hospitalization. Therefore, there is a higher probability for them to be involved in accidents

Copyright ( 2016 Chen Li and Chen Xiao

doi: $10.18686 /$ jn.v5i3.8

Received: June 21, 2016; Accepted: August 6, 2016; Published online: August 24, 2016

This is an open-access article distributed under the terms of the Creative Commons Attribution Unported License (http://creativecommons.org/ licenses/by-nc/4.0/), which permits unrestricted use, distribution, and reproduction in any medium, provided the original work is properly cited.

${ }^{\star}$ Corresponding author: Hospital of No. 8740 People's Armed Force, Nanchong, Sichuan 637000, China. E-mail: chenli1235@sina.com especially accidental falling [2]. Many factors contribute to the accidental falling of the psychotic inpatients and can be summarized into two categories: First, patients' own factors; second, the hospital environmental factors [3]. This paper studies and analyzes the factors that cause the falling of the psychotic inpatients and prevention method taken in order to protect them from the risk of falling that lead to relevant danger.

\section{Data and Methods}

\subsection{General data}

100 psychotic patients are selected. These patients have experienced accidental falling during hospitalization in our hospital from June 2012 to June 2014. Among them, there are 67 male patients is and 33 female patients. Their age varies from 23 to 56 and their average age is (32.5 \pm 9.5). The duration of the hospitalization of these patients ranges from 1 to 12 months and the average hospitalization duration is $(5.0 \pm 2.5)$ months. 45 of these patients have schizophrenia, 45 have affective disorders and 10 have Alzheimer Disease. The medications currently used by these patients mainly include: Chloropropanols, clonazepam and risperidone, etc. and all selected patients are in compliance with the Standards for Clinical Diagno- 
sis of Geriatric Psychiatry stipulated in the $3^{\text {rd }}$ edition of "Chinese Standards for Classification and Diagnosis of Mental Disorders". Besides, the patients and their families are well informed of the treatment methods and nursing measures, etc. and they are willing to sign the Informed Consent Form. There are no significant differences between the patients in terms of gender and age $(p>0.05)$, therefore, they are comparable.

\subsection{Research methods}

The percentage of the falling caused by the patients' own factors and hospital environmental factors separately are studied. In the meantime, the venues where the fall happen and the time when the fall happen are recorded. Therein, the patients' own factors include disease factor; poor selfcare ability; epileptic attack; side effects off drug while the hospital environmental factors include: ground environment; lighting environment.

\subsection{Observation items}

The observation items are the time of the psychotic patients' accidental falling and the places where the accidental falling happen.

The time are divided into three periods: Daytime (8:00 a.m. to 16:00 p.m.), night time (16:00 p.m. to 00:00 a.m) and midnight (00:00 a.m to 8:00 a.m.).

The places are: Wards, corridors and toilets.

\subsection{Statistical methods}

The collected data are analyzed with SPSS 18.0 software. Therein, the data in conformity with the Gaussian distribution are processed with single factor analysis of variance and if there is statistical significance, then it will be handled with one-to-one comparison with LSD method. $p<0.05$ suggests there are differences that are statistically significant between the two sets of data by comparison.

\section{Result}

\subsection{Analysis of the factors causing the falling of the patients}

The findings of the analysis of the factors that caused the falling of the patients are as follows: Among the cases of falling of the psychotic inpatients, $82.0 \%$ $(82 / 100)$ of the cases are caused by the patients' own factors and $18.0 \%(18 / 100)$ of the cases are caused by the hospital environmental factors. Among the factors related to the patients themselves, disease factor plays a dominant role by causing $(34.0 \%)$ of the falling and the side effects of drug plays a secondary role. Among the hospital environmental factors, the ground environment takes the first place in terms of importance by causing (14.0\%) of the falls (Table 1$)$. The patients' own factors cause far more falling than the hospital environmental factors.
Table 1. Comparison of factors causing the falling of the patients.

\begin{tabular}{ccc}
\hline Factor & Cases & Percentage \\
\hline Patients' own factors & 82 & $82.0 \%$ \\
Disease & 34 & $34.0 \%$ \\
Poor self-care ability & 10 & $10.0 \%$ \\
Epileptic attack & 8 & $8.0 \%$ \\
Side effects of drug & 30 & $30.0 \%$ \\
Hospital environmental factors & 18 & $18.0 \%$ \\
Ground environment & 14 & $14.0 \%$ \\
Lighting environment & 4 & $4.0 \%$ \\
\hline
\end{tabular}

\subsection{Comparison of the time of the patients' falling} 18 patients tumbled in daytime, 23 patients fell in the evening and 59 in the midnight period. For details, see Table 2.

Table 2. Comparison of the time of the patients' falling (case\%).

\begin{tabular}{cccc}
\hline Time of tumble & Daytime & Night time & Midnight \\
\hline Cases $(n=100)$ & $18(18.0)$ & $23(23.0)$ & $59(59.0)$ \\
\hline
\end{tabular}

It can be seen from Table 2 that the falling of the psychotic inpatients mostly occurred in the midnight period.

\subsection{Comparison of the places of the patients' falling}

20 patients fell in the wards, 26 in the corridors and 54 in the toilets (Table 3). It can be seen from Table 3 that most of the falling of the psychotic inpatients occurred in the toilets.

Table 3. Comparison of places of the patients' falling (case\%).

\begin{tabular}{cccc}
\hline Place of tumble & Wards & Corridors & Toilets \\
\hline Cases $(n=100)$ & $20(20.0)$ & $26(26.0)$ & $54(54.0)$ \\
\hline
\end{tabular}

\section{Discussion}

Accidental falling often occurs in psychotic inpatients due to their mental state, self-care ability and other factors. In severe cases, accidental falling can lead to bone fractures or brain damage of the patients and can even lead to death sometimes. Accidental falling does not only affect the rehabilitation of patients, but also impose a negative impact on the communication between doctors and patients [4].

According to the analysis of the factors causing the accidental fall of the patients, $82.0 \%(82 / 100)$ of the accidental falls of the psychotic inpatients are caused by the factors related to the patients themselves and $18.0 \%(18 / 100)$ of the accidental falls are caused by the hospital environment. Among the factors related to the patients, the disease factor causes the most accidental falls (34.0\%)and the side effects of drug causes the second most common cause of accidental falls, which is according to the roles the factors play in causing the accidental falls, disease factor ranks No. 1 and side effects of the drug ranks No. 2. Among the hospital 
environmental factors, according to the roles they play in causing the accidental fallings, the ground environment ranks $1^{\text {st }}$ by causing (14.0\%) of the accidental falls. Thus, it can be seen that the factors of the patients per se cause a majority of the fall and among them, the disease factor and side effects of the drug are the reasons for most of the fall. Apart from that, the hospital environmental factors also play an important role in causing the falls. The side effects of the drug refer to the orthostatic hypotension or dizziness induced by drug. As for the hospital environment, the damp ground and dim interior light pose the greatest risk for the fall of the inpatients [5,7].

Because self-care ability of the patients with mental illness is far lower than that of the general hospitalized patients, it is necessary to strengthen the hardware and software nursing of the psychotic inpatients during their hospitalization period. In the aspect of hardware which is in the indoor areas where the patients visit frequently, the ground should be kept dry to prevent the patients from slipping. The high-risk areas like toilets and corridors should be equipped with handrails and armrests for the patients' convenience. At the same time, the indoor lighting should be adequate so that the eyesight of the patients in the evening and at midnight will be clear. In the aspect of software, it is necessary to strengthen the professional training of the nursing staff about nursing of psychotic patients and to strengthen observation of the psychotic patients during nursing. When guiding the patients to take drugs, it is necessary to give them precautions to avoid the dizziness induced by the side effects of the drugs and to avoid the fallings induced by the dizziness.

The time factor also matters a lot to the occurrence of accidental fallings. The relevant data shows that the accidental falling of the patients happen within two time periods, that is, the period from 16:00 p.m. to 21:00 p.m. and the period from 21:00 p.m. to 8:00 a.m. In the first period, because the various basic treatments is finished, the elderly patients have more time to get off the bed and walk around, which increases the occurrence rate of the falling; in the second period, the elderly patients, especially the male patients need to answer nature's call quite frequently, making them prone to falling. Meanwhile, in the early morning, most of the patients went to toilet for their daily routine activities which makes it more possible for the falling cases to happen. To better ensure the patients' safety, medical staff should inform patients' family regarding the patient's condition therefore it is better for them to accompany and monitor the patients 24 hours to eliminate potential safety hazards [6].

In addition, environmental factors also affect the accidental falling of the hospitalized psychotic patients. The relevant data demonstrates that there is a close relationship between the fallings of the elderly people aged 65 or above and the environment they are put in. In details, the insufficient indoor lighting, slippery ground and improper shoes the patients wear, the lack of safety rails in the places where the patients move around, the improper height of the bed and the absence of window frame, etc., together with the unfamiliarity of the patients with the surrounding environment, make it easier for patients to fall down. Therefore, when nursing the patients with mental illnesses in clinical practice, it is necessary to take appropriate preventive measures and focus on protection against the high-risk factors that cause the patient's falling to reduce their accidental falls to the maximal degree and ensure the speedy recovery of the patients [8].

In summary, during the nursing to prevent the falling of the psychotic inpatients, it is necessary to strengthen the monitoring of their illness conditions, improve the nurses' awareness of the importance of the life nursing to the psychotic patients, and to equipped the places where the psychotic patients live and visit frequently with protective facilities.

\section{Conflicts of interest}

These authors have no conflicts of interest to declare.

\section{Authors' contributions}

These authors contributed equally to this work.

\section{References}

1. Wang L, Li Y, Liu G. Analysis of the risk factors causing the fallings of the geriatric patients and the nursing intervention of the peri-operative period. Med Forum. 2013;17(18):2313-2315.

2. Su S. Application of process management in prevention of the fallings of the geriatric inpatients of the neurology department. World Latest Med Inf. 2013;13(7):475-478.

3. Chen T, Tao X, Duanmu X. Analysis of multiple factors for fallings of the hospitalized mental patients. Int $J$ Nurs. 2010;(11):1657-1659.

4. Wang X, Liu X, Liang Y. Application of falling-causing risk factor evaluation to geriatric patients with lumbar degenerative disease. J Nurs Adm. 2014;14(6):433-434.

5. Liang $F, H u X$. Investigation of the tumble conditions of the psychiatric inpatients and management strategies. Med J Chin People's Health. 2012;24(21):2675-2677.

6. Lu Z, Guan W. Analysis of the effects of the health education given to the psychiatric inpatients to prevent fallings. Med J Chin People's Health. 2011;23(6):760-761.

7. Gao Y. Risk factors for the fallings of the geriatric patients with mental illness and intervention measures. Med J Chin People's Health. 2010;22(6):731-732.

8. Lu S, Huang Q, Xu Y, et al. The analysis of the root causes of the fallings of the geriatric inpatients and countermeasures. Chin J Clin Health Care. 2011;14(4):422. 